\title{
Stage IVA Hilar Cholangiocarcinoma AJCC v8
}

National Cancer Institute

\section{Source}

National Cancer Institute. Stage IVA Hilar Cholangiocarcinoma A/CC v8. NCI Thesaurus. Code C134752.

Stage IVA includes: Any T, N2, M0. N2: Four or more positive lymph nodes involving the hilar, cystic duct, common bile duct, hepatic artery, posterior pancreatoduodenal, and portal vein lymph nodes. M0: No distant metastasis. (AJCC 8th ed.) 